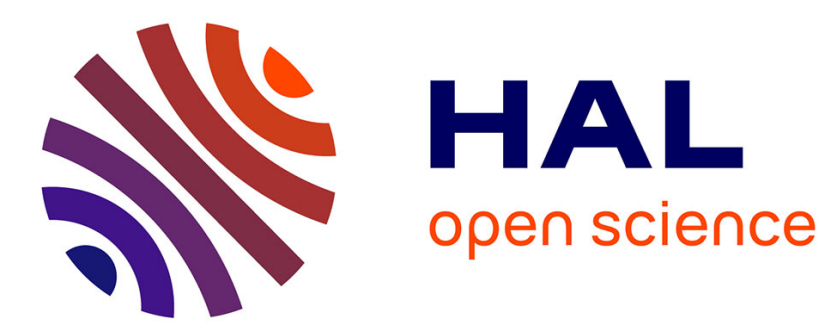

\title{
Structured adaptive attitude control applied on a Myriade simulation benchmark
}

Alexandru-Razvan Luzi, Jean-Marc Biannic, Jean Mignot, Dimitri Peaucelle

\section{To cite this version:}

Alexandru-Razvan Luzi, Jean-Marc Biannic, Jean Mignot, Dimitri Peaucelle. Structured adaptive attitude control applied on a Myriade simulation benchmark. AIAA Guidance Navigation \& Control Conference, Aug 2013, BOSTON, United States. hal-01058619

\section{HAL Id: hal-01058619 \\ https://hal-onera.archives-ouvertes.fr/hal-01058619}

Submitted on 27 Aug 2014

HAL is a multi-disciplinary open access archive for the deposit and dissemination of scientific research documents, whether they are published or not. The documents may come from teaching and research institutions in France or abroad, or from public or private research centers.
L'archive ouverte pluridisciplinaire HAL, est destinée au dépôt et à la diffusion de documents scientifiques de niveau recherche, publiés ou non, émanant des établissements d'enseignement et de recherche français ou étrangers, des laboratoires publics ou privés. 


\title{
Structured adaptive attitude control applied on a Myriade simulation benchmark
}

\author{
Alexandru-Razvan Luzi and Jean-Marc Biannic \\ ONERA, The French Aerospace Lab, 2 avenue Edouard Belin, F-31055 Toulouse, France \\ CNRS, LAAS, 7 avenue du Colonel Roche, F-314000 Toulouse, France \\ Jean Mignot \\ CNES ; DCT/SB/PS ; BPI 1712, 18 avenue E. Belin, F-31401 Toulouse, France \\ Dimitri Peaucelle \\ CNRS, LAAS, 7 avenue du Colonel Roche, F-31400 Toulouse, France \\ Univ. de Toulouse, LAAS, F-31400 Toulouse, France
}

\begin{abstract}
This paper considers the problem of reaction-wheel attitude control inside the mission mode of the Myriade satellites. A structured adaptive algorithm, allowing to extend the operating domain of a static proportionalderivative controller is presented and conditions for designing a stabilizing, continuous-time adaptive law are given. In view of implementation, a discrete-time adaptive algorithm is derived and tested on a benchmark of the DEMETER satellite, which was part of the Myriade program. Simulation results show that the structured adaptation and the use of the $\sigma$-modification allow the adaptive closed-loop to follow attitude step references of up to 20 degrees without saturating the reaction wheels. This allows the adaptive law to cover the whole mission mode and replace the currently-implemented switched-based control strategy, thus potentially simplifying the verification and validation. process.
\end{abstract}

\section{Introduction}

The Centre National dEtudes Spatiales (CNES) Myriade series is a concept of modular microsatellites weighing less than $200 \mathrm{~kg}$ that afford quick, low-cost access to space. The aim of Myriade is to bring space research within easier reach of the scientific community, particularly for scientists working on large-scale international missions and facing long lead times between the planning and implementation stages. DEMETER (detection of electromagnetic emissions transmitted from earthquake regions), ${ }^{1,2}$ the first microsatellite of the Myriade series, was launched in 2004 and was taken off service in 2011. Its complete model of DEMETER is now open for the scientific community ${ }^{3}$ and will be used in this paper as a benchmark. Two other missions, Parasol and Picard are still orbiting and the two next one Taranis and Microscope will be launched respectively in 2015 and 2016. The evolutions of the Myriade series was intend to enhance the payload capability. The flight domain has been redefined in order to cope with the probable missions over this period: Earth observation and monitoring, sun and space observation (from Low Earth Orbit (LEO)), defense program. These led to specify a range of $500-800 \mathrm{~km}$ altitude, Sun Synchronous Orbit (SSO) orbits for all Local Time at Ascending Node (LTAN). The specified in orbit lifetime is 5 years, with extended qualification to 7 years when possible.

The on-orbit life of the Myriade satellites is decomposed, as for all satellites in general, into phases, allowing the convergence from the separation state to the mission state, the orbit control, and the safe state in case of fault. For each phase or mode, a specific set of attitude pointing requirements is to be met by using a specific set of actuators and sensors. These requirements define the associated Attitude Control mode. Inside a mode, several attitude control and estimation algorithms can be designed, in order to meet increasing performance levels requirements. Over the past thirty years, the number of Attitude and Orbit Control System (AOCS) modes has been reduced, so that generic architectures now typically consist of three modes (safehold, mission and orbit control mode). In the same time the software complexity has increased to meet ever more demanding requirements. Thus, the switching between different AOCS modes requires exhaustive verification and validation in order to demonstrate the robustness of the system with stringent kinematics conditions and switching of attitude control sensors. 
In this paper, the problem of attitude control inside the mission mode of the Myriade satellites is considered. Due to the limitations of the reaction wheels used for actuation, the use of a single LTI controller over the whole flight domain covered in the mission mode would not be adequate: the controller would either demand huge actuator solicitation at large pointing errors or generate a very slow closed-loop response. Therefore, the desired closed loop behaviour can only be obtained with a varying controller : when the pointing error is large the controller has to ensure a response that is not over-demanding for the actuators, while a rapid and precise response is required when the pointing error is smaller. The existing controller of the Myriade satellites satisfies this demand by switching between two control laws at large pointing errors a speed tracking loop (momentum bias) is used and at small angles the controller switches to an attitude proportional- derivative law. A satisfying response time and a limited control activity (in particular a limitation of the reaction wheel rotation speed and a null torque for transient regimes, once the travelling speed is reached) are thus ensured. A discontinuity in the control torque can however appear if this travelling speed has not been reached at the switching point. The switch also induces abrupt changes in the closed-loop plant and makes the stability analysis difficult. Based on these remarks, the replacement of the switched-based strategy by a direct adaptive control law is proposed and validated by simulations.

This work was induced by previous studies dealing with the application of robust control techniques to space activities. The suitability of such new techniques has been proven for very various space applications, including the use and validation of robust techniques for the attitude control of Demeter with his flexible appendixes. ${ }^{4-6}$

This paper considers the direct adaptive control scheme, ${ }^{7,8}$ consisting of directly tuning the controller gains, based solely on the measured outputs of the system. With respect to more complex estimation/gain scheduling scheme, it has the advantage of simplicity and for this reason it is also referred to as simple adaptive control. ${ }^{8}$ As this scheme is often based on passivity properties, it is equally called passivity-based adaptive control. ${ }^{9,10}$

The considered adaptive output feedback law writes, in the general cas, $u(t)=K(t) e(t)$, where $e(t)=y(t)-y_{r}(t)$ is the error with respect to a reference signal $y_{r}$ and where $K(t)$ is the adaptive gain. In this paper, gain and output structuring is considered, such that $u(t)=\sum_{i=1}^{\bar{\imath}} K_{i}(t) e_{i}(t), e_{i}(t)=y_{i}(t)-y_{r i}(t)$, where each gain component $K_{i}(t)$ is adapted independently, according to a differential equation. This equation contains a gradient-type term $-G_{i} e e_{i}^{T}$, which drives the gains to stabilizing values, according to passivity properties. The use of different $G_{i}$ matrices is inspired by Ref. 11 and is an extension of the $G$-passivity property introduced in Ref. 12. The choice of $e_{i}$ and constraints that can be imposed on $G_{i}$ offer important degrees of freedom in designing the adaptive law and prove to be particularly useful for satellite attitude control. The second term of the adaptation equation is a barrier term $-\Phi\left(K_{i}-F_{0 i}\right)$, that keeps the gains inside a bounded domain. A term of the type $-\sigma_{i}\left(K_{i}-F_{0 i}\right)$ (usually called $\sigma$-modification $\left.{ }^{8}\right)$ is also considered. This term is generally used ${ }^{13}$ for persistent noise compensation as well as for driving the adaptive gains to the "nominal" values $F_{0 i}$, when the tracking errors become weak.

Structuring the adaptation and the use of the $\sigma$-modification allows, for the considered application, obtaining a controller with an enlarged operating domain. In this way a single adaptive algorithm can be applied over the whole mission mode of the Myriade satellites.

This paper is organized as follows. First, a section describes the Myriade attitude control problem and the DEMETER benchmark. Section 3 presents the method allowing to design stabilizing continuous-time structured adaptive laws, and presents a possibility for deriving a discrete-time law, suitable for implementation on on-board computers. The discrete adaptive law is applied in Section 4 on the DEMETER benchmark and the simulation results are given. Finally, some concluding remarks are driven.

\section{Notations}

$\mathrm{R}^{m \times p}$ is the set of real $m$ by $p$ matrices. $A^{T}$ is the transpose of the matrix $A . \operatorname{Tr}(A)$ is the trace of $A . \mathbf{1}$ and $\mathbf{0}$ are respectively the identity and the zero matrices of appropriate dimensions. For symmetric matrices, $A>(\geq) B$ means that $A-B$ is positive (semi) definite. For a non symmetric matrix $\{A\}^{\mathcal{S}}=A+A^{T} .\|A\|_{D}=\sqrt{\operatorname{Tr}\left(A^{T} D A\right)}$ is the weighted Frobenius norm of A with weight $D>\mathbf{0}$.

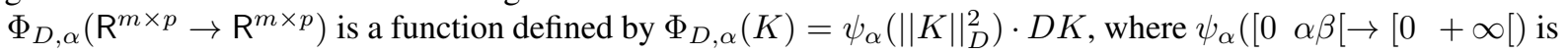
a scalar function having the expression of a dead-zone around the origin $\psi(0 \leq k \leq \alpha)=0$ and that of an increasing barrier going to infinity as $k$ tends to $\alpha \beta$. An example of such a function is $\psi(\alpha \leq k<\alpha \beta)=(k-\alpha) /(\alpha \beta-k)$.

\section{Myriade - Attitude control}

This section briefly presents the AOCS control loop of the Myriade satellites, with an emphasis on the currently implemented switching-based controller, represented in Fig. 1. Models of the DEMETER satellite (see ${ }^{3}$ for a full full 
description of the DEMETER benchmark), which was part of the Myriade program, are used.

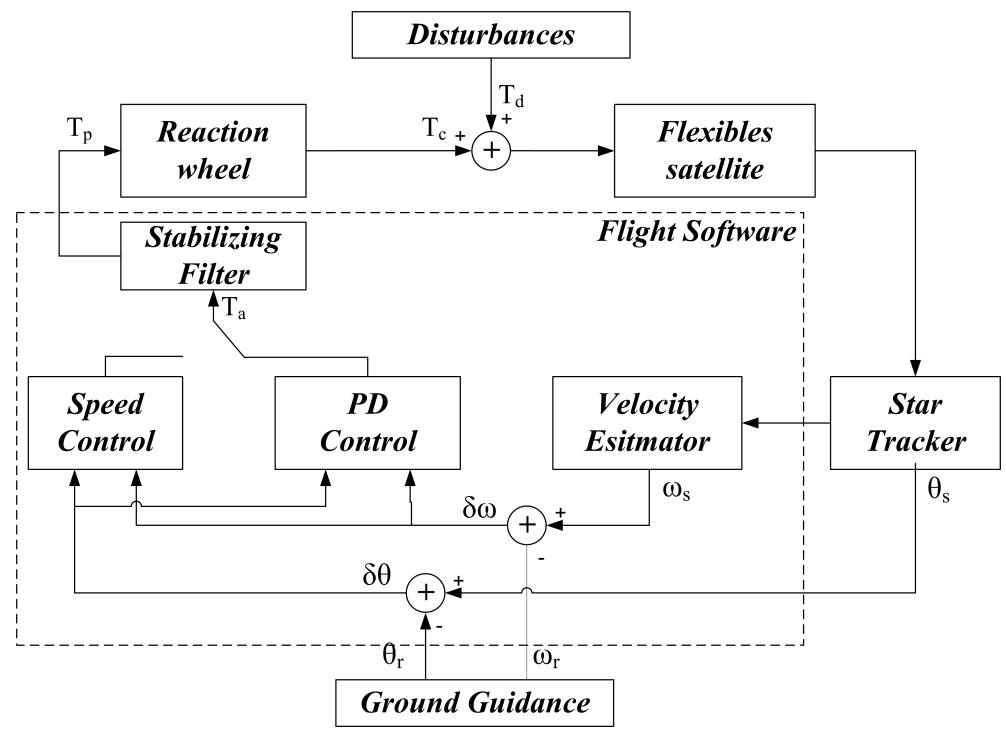

Figure 1. Myriade AOCS loop

\section{II.A. Flexible satellite model}

The satellite dynamics are represented by a 3 inputs - 3 outputs LTI model containing four pairs of flexible modes: ${ }^{3}$

$$
\left[\begin{array}{cc}
J & J_{f} \\
J_{f}^{T} & \mathbf{1}
\end{array}\right]\left[\begin{array}{c}
\ddot{\delta \theta} \\
\ddot{\eta}
\end{array}\right]=\left[\begin{array}{cc}
\mathbf{0} & \mathbf{0} \\
\mathbf{0} & -C_{S}
\end{array}\right]\left[\begin{array}{c}
\dot{\delta \theta} \\
\dot{\eta}
\end{array}\right]+\left[\begin{array}{cc}
\mathbf{0} & \mathbf{0} \\
\mathbf{0} & -K_{S}
\end{array}\right]\left[\begin{array}{c}
\delta \theta \\
\eta
\end{array}\right]+\left[\begin{array}{l}
\mathbf{1} \\
\mathbf{0}
\end{array}\right] T_{C}
$$

where $\delta \theta \in \mathrm{R}^{3}$ is the vector of angular deviations from a reference $\delta \theta=\theta-\theta_{r}$ around the axes $\mathbf{X}, \mathbf{y}, \mathbf{z}$ of the satellite; $\eta \in \mathrm{R}^{8}$ is the state vector associated to the flexible modes (two states per flexible mode). $J$ and $J_{f}$ are respectively the axes inertia and the coupling matrix between $\delta \theta$ and the flexible modes, composing the generalized mass matrix. Off-diagonal terms of $J$ are in first approximation negligible making the three axes dynamics almost decoupled. The matrices

$$
C_{s}=\operatorname{diag}_{k=1 \ldots 4}\left(\left[\begin{array}{cc}
2 \zeta_{k} \omega_{k} & 0 \\
0 & 2 \zeta_{k} \omega_{k}
\end{array}\right]\right), K_{s}=\operatorname{diag}_{k=1 \ldots 4}\left(\left[\begin{array}{cc}
\omega_{k}^{2} & 0 \\
0 & \omega_{k}^{2}
\end{array}\right]\right)
$$

are diagonal generalized matrices of damping and stiffness respectively. ${ }^{14} T_{c}$ is the control torque applied to the satellite.

The synthesis models are given by SISO representations of each axis (the inter-axes coupling is thus neglected at the control design stage). The inertias are chosen equal to the nominal value and one flexible mode is considered, defined by $\omega_{1}=0.4 \cdot 2 \pi \mathrm{rad} / \mathrm{s}$ and $\zeta_{1}=2.7 \cdot 10^{-3}$ (the next flexible modes have less influence and are neglected for the control design). For illustration, the expression of the associated transfer function for the $\mathrm{x}$ axis is given:

$$
H_{\text {sat }, \mathrm{x}}(s)=\frac{0.03933 s^{2}+0.0005437 s+0.2485}{s^{4}+0.01706 s^{3}+7.797 s^{2}}
$$

Once the design of adaptive controllers done on these simplified satellite models, the control law is tested of the 3-axes models with all four flexible modes defined by :

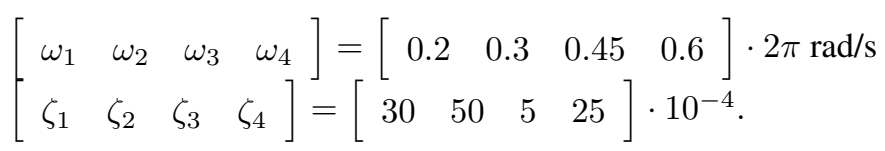

Notice that the natural frequency and damping of the first flexible mode is different in simulation from the one used at the design stage. This contributes to testing robustness. 


\section{II.B. Actuator and sensor model}

The controlled torque for each axis is delivered by accelerating a reaction wheel. Its model is given in Fig. $2^{3}$ :

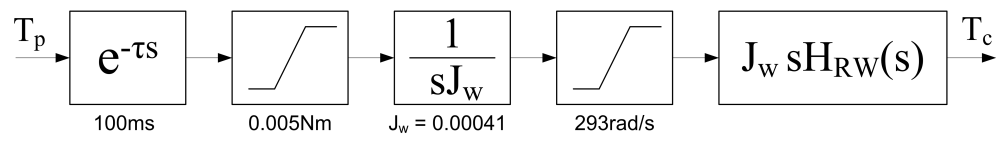

Figure 2. Reaction wheel model

with:

$$
H_{\mathrm{RW}}(s)=\frac{1.214 s+0.7625}{s^{2}+2.40 s+0.7625}
$$

Two saturation blocks can be observed in this model. The one on the rotation speed is critical: if the wheel reaches the maximal speed then the generated torque is zero. The satellite becomes non-controllable, which is critical, as it is open-loop unstable (double integrator). The wheel model used for the controller synthesis is given by $H_{\mathrm{RW}}(s)$. The control law is then tested in simulation with the complete model represented in Fig. 2.

The attitude measure is given by a star tracker, modelled in simulation by a 0.45 seconds delay and an additive noise. For the synthesis model, this measure is considered perfect. The delay corresponding to the reaction wheel and the star tracker could be taken into consideration for synthesis by the means of Pade approximations. This is not done here, the synthesis model assuming zero delays. Robustness with respect to these neglected dynamics is then tested by simulation, where all experiments are done including the delays.

\section{II.C. Existing flight software}

The attitude control is achieved in a decentralized way, each axis being controlled independently. For each axis, the flight software is composed of an angular velocity estimator and a switching control law followed by a stabilizing linear filter.

The satellite angular velocities are estimated from the angular positions via a high pass filter defined by the transfer function $H_{\text {estim }}(s)=\frac{s}{1+0.5 s}$.

For each axis, the reaction wheel control system consists of a nonlinear law for large pointing errors which switches to a proportional-derivative controller when the pointing error becomes small:

$$
\left\{\begin{array}{l}
T_{a \mathrm{i}}=-k_{0}\left(\delta \omega_{\mathrm{i}}+\omega_{d} \operatorname{sign}\left(\delta \theta_{\mathrm{i}}\right)\right), \quad \text { if }\left|\delta \theta_{\mathrm{i}}\right|>\theta_{L} \\
T_{a \mathrm{i}}=-\left(F_{0 \theta \mathrm{i}} \delta \theta_{\mathrm{i}}+F_{0 \omega \mathrm{i}} \delta \omega_{\mathrm{i}}\right), \quad \text { if }\left|\delta \theta_{\mathrm{i}}\right| \leq \theta_{L}
\end{array}\right.
$$

where $\delta \theta_{\mathrm{i}}$ is the pointing error around axis $\mathrm{i} \in\{\mathrm{x}, \mathrm{y}, \mathrm{z}\}$.

The threshold $\theta_{L}$ and speed reference $\omega_{d}$ allow to optimize a trade-off between convergence time and control activity. They are chosen the same for all axes. For DEMETER, the numerical values are: $\omega_{d}=0.015 \mathrm{deg} / \mathrm{s}$ and $\theta_{L}=0.3 \mathrm{deg}$ and the scalar parameter $k_{0}=1$. The gains $F_{0 \theta \mathrm{i}}$ and $F_{0 \omega \mathrm{i}}$ are chosen so that the produced torque is null, when the reference speed has been reached, and continuous at the switching point. This constraint implies:

$$
F_{0 \theta i} \theta_{L}=F_{0 \omega i} \omega_{d}
$$

and the numerical values are: $F_{0 \theta \mathrm{i}}=F_{0 \theta}=0.1$ and $F_{0 \omega \mathrm{i}}=F_{0 \omega}=2$ for all three axes $\mathrm{i} \in\{\mathrm{x}, \mathrm{y}, \mathrm{z}\}$. This design generally guarantees the non-saturation of the reaction wheel, that of its speed in particular.

In order to improve stability and to guarantee a good precision level at low pointing errors, a linear filter has been added after the switching structure. ${ }^{4}$ For the $\mathrm{x}$ satellite axis, it is given by the transfer function:

$$
H_{\text {filter }, \times}(s)=\frac{3.039 s^{2}+1.457 s+0.09635}{0.3333 s^{4}+1.371 s^{3}+1.263 s^{2}+0.4489 s}
$$

These filters (one per axis) are designed taking into account flexible modes, to satisfy an $H_{2} / H_{\infty}$ trade-off of the linear model close to the equilibrium $\left(|\delta \theta| \leq \theta_{L}\right)$. For small pointing errors $\left(\theta_{L}=0.3 \mathrm{deg}\right)$, the decentralized control $T_{p \mathrm{i}}=-H_{\text {filter,i }}(s)\left(F_{0 \theta} \delta \theta_{\mathrm{i}}+F_{0 \omega} \delta \omega_{\mathrm{i}}\right)$ can therefore be considered as optimal.

The control algorithm runs on the on-board computer at a frequency of $4 \mathrm{~Hz}$ and discrete representations of the stabilizing filters are obtained from the corresponding continuous-time transfer functions by using the bilinear transformation. 


\section{II.D. Towards adaptive control}

Even though the existing switching structure answers most control objectives (fair system response time, no actuator saturation, good robustness properties) it has however several drawbacks: the first one regards the possible discontinuity in the computed torque at the switching point. This can happen for instance when switching from the coarse to the fine pointing mode, if the reference speed has not been reached. Also, if this law needs to be modified, several parameters need to be changed at the same time, in order to verify the continuity condition (7). For instance, if the gain $F_{0 \theta}$ needs to be changed, then either $\theta_{L}$ or $\omega_{d}$ have to be modified. Redesign can therefore prove to be delicate.

These are the main reasons for which adaptive control is investigated in this paper. One of the advantages of using such a strategy, is to have a control law covering a larger functioning domain. This would allow replacing the switching-based strategy and potentially simplify the validation process: only one control law would have to be validated instead of two, as in the case of the switching strategy. Adaptive algorithms also have the advantage of gains that vary continuously in time, thus avoiding the switching related problems. Finally changing the parameters of an adaptive algorithm that is unique over the whole operating domain will impact the global behaviour of the system. This would allow, for instance, the improvement of the dynamical performances.

Based on these considerations, an adaptive law based on the proportional-derivative controller is proposed. For each axis $i \in\{x, y, z\}$ this law reads as:

$$
T_{p \mathrm{i}}(t)=-H_{\text {filter }, \mathrm{i}}(s)\left(K_{\theta \mathrm{i}}(t) \delta \theta_{\mathrm{i}}(t)+K_{\omega \mathrm{i}}(t) \delta \omega_{\mathrm{i}}(t)\right)
$$

where the gains $K_{\mathrm{i}}(t)=\left[\begin{array}{ll}K_{\theta \mathrm{i}}(t) & K_{\omega \mathrm{i}}(t)\end{array}\right]$ are adapted around the values $F_{0}=\left[\begin{array}{ll}F_{0 \theta} & F_{0 \omega}\end{array}\right]=\left[\begin{array}{ll}0.1 & 2\end{array}\right]$ known to be optimal for small pointing errors.

When designing the adaptation rules for the gains $K_{\theta \mathrm{i}}(t)$ and $K_{\omega \mathrm{i}}(t)$, a key issues is to into account the constraints regarding avoidance of reaction wheel saturations, that of its angular speed in particular. Standard approaches to cope with such actuators limitations are based on anti-windup design techniques. ${ }^{15-17}$ The central idea behind these techniques - initially developed in the early 1960's and extensively revisited in the recent literature - consists of synthesizing a controller augmentation that has no effect when saturation does not occur and that otherwise attempts to provide satisfactory performances despite possibly large or fast varying control input signals. The anti-windup device can then be viewed as an additional loop which is designed a posteriori to modify the nominal control laws when saturations appear in the closed-loop system.

The adopted strategy is somehow related but indirect. It is based on the following facts.

- At small pointing error the gains $F_{0}=\left[\begin{array}{ll}F_{0 \theta} & F_{0 \omega}\end{array}\right]=\left[\begin{array}{ll}0.1 & 2\end{array}\right]$ are chosen for fast compensation of perturbations. If applied at large pointing error $\left(\left|\delta \theta_{\mathrm{i}}\right|\right.$ large $)$ the first term gives a value that goes beyond torque saturation (and would hence, also generate the wheel speed saturation). In term of adaptation rules, this indicates that for large pointing error $K_{\theta \mathrm{i}}(t)$ should be decreased such that the possibility of saturating the reaction wheels is reduced. When $\left|\delta \theta_{\mathrm{i}}\right|$ becomes small, $K_{\theta \mathrm{i}}(t)$ should return the nominal value $F_{0 \theta}$. Note that in order to deal with large pointing errors, $K_{\theta \mathrm{i}}(t)$ should be allowed to take values as small as possible. In other words, the domain inside which the gain is allowed to evolve should be as large as possible.

- With respect to the gains $K_{\omega \mathrm{i}}(t)$, the physics indicate that globally the speed of reaction wheels follows the angular speed of the satellite. The errors on the angular speed of the satellite should therefore be kept as small as possible to avoid the speed of reactions wheels to saturate. This imposes to choose $\delta \omega_{i}=\omega_{i}-\omega_{r i}$ with $\omega_{r \mathrm{i}}=0$ and have $K_{\omega \mathrm{i}}(t)$ increase when $\left|\delta \omega_{\mathrm{i}}\right|$ is large. When $\left|\delta \omega_{\mathrm{i}}\right|$ becomes small, $K_{\omega \mathrm{i}}(t)$ should return to the value $F_{0 \omega}$ such that the nominal behaviour is kept.

\section{Structured adaptive control}

This section gives the theoretical results necessary for designing adaptive laws satisfying the constraints of the considered attitude problem. Conditions for obtaining a stabilizing continuous-time adaptive law are given. The continuous law is then sampled in order to obtain a discrete algorithm suitable for implementation on the satellite computer.

The following systems are considered:

$$
\left\{\begin{array}{l}
\dot{x}=A x+B u \\
y=C x=\left[\begin{array}{lll}
C_{1}^{T} & \ldots & C_{\bar{\jmath}}^{T}
\end{array}\right]^{T} x=\left[\begin{array}{lll}
y_{1}^{T} & \ldots & y_{\bar{\jmath}}^{T}
\end{array}\right]^{T}
\end{array}\right.
$$


where $x \in \mathrm{R}^{n}$ is the state, $u \in \mathrm{R}^{m}$ the control input vector and $y \in \mathrm{R}^{p}$ the measured output, structured in sub-vectors $y_{j} \in \mathrm{R}^{p_{j}}$, with $y_{j}=C_{j} x, j=1 \ldots \bar{\jmath}$. $\jmath$ is the number of sub-vectors in which the output is divided and it can be chosen by the designer.

The existence of a stabilizing static output feedback law is assumed:

$$
u=F_{0} e=\sum_{i=j}^{\bar{j}} F_{0 j} e_{j}
$$

where $F_{0}=\left[\begin{array}{lll}F_{01} & \ldots & F_{0 \bar{\jmath}}\end{array}\right]$ is the stabilizing static output feedback and each $F_{0 j}$ is the gain component corresponding to $e_{j} . y_{r}$ represents the reference, $e=y-y_{r}$ the tracking error and vectors $y_{r}$ and $e$ are structured in a similar manner to the output: $e_{j}=y_{j}-y_{r_{j}}, j=1 \ldots \bar{j}$. $F_{0}$ guarantees stability of the closed loop state matrix: $A\left(F_{0}\right)=A+B F_{0} C$.

\section{III.A. Structured adaptive control}

When considering the attitude control problem for one axis models of the satellite, the static law $u(t)=F_{0} e(t)$ corresponds to the proportional-derivative controller (6) $\left(e=\left[\begin{array}{ll}\delta \theta & \delta \omega\end{array}\right]^{T}\right)$, which is optimal when the system is closed to the equilibrium point, but has a limited operating domain $(|\delta \theta| \leq 0.3 \mathrm{deg})$. Starting from this static law, $u(t)=$ $F_{0} e(t)$, we aim at obtaining an adaptive algorithm, $u(t)=K(t) e(t)$, which could cover an extended operating domain.

The considered adaptive control is of the following type:

$$
\left\{\begin{array}{l}
\dot{K}_{j}(t)=-G_{j} e(t) e_{j}(t)^{T} \Gamma_{j}-\Phi_{D_{j}, \alpha_{j}}\left(K_{j}(t)-F_{0 j}\right) \Gamma_{j} \\
u(t)=K(t) e(t)=\sum_{j=1}^{\bar{j}} K_{j}(t) e_{j}(t)
\end{array}, j=1 \ldots \bar{\jmath}\right.
$$

It is structured as follows. The control action is decomposed in a sum of $K_{j}(t) e_{j}(t)$ adaptive feedbacks with the aim of tuning separately each of the related adaptations laws. This allows to include engineering knowledge about the process to stabilize. It can for example allow to have fast adaptation with respect to errors measured via $e_{1}$ and slower adaptation with respect to the other errors. More than just the speed of adaptation (which is tuned via the positive definite gains $\Gamma_{j}$ ), each adaptation law can be given a 'direction' by the choice of matrices $G_{j}$. Imposing $G_{1} e=g_{1} e_{1}$ where $g_{1}$ is a positive scalar will force $K_{1}$ to decrease when $e_{1} e_{1}^{T}$ is large (go for lower gain when the error is large). Imposing $G_{1} e=-g_{1} e_{1}$ will on the contrary force $K_{1}$ to grow.

As in the case of attitude control, for one-axis models, $e \equiv\left[\begin{array}{ll}\delta \theta & \delta \omega\end{array}\right]^{T}$, with $e_{1} \equiv \delta \theta$ and $e_{2} \equiv \delta \omega, \bar{\jmath}=2$, the proposed structure proves to be valuable for this application. Indeed, the requirements regarding the gains evolution, presented in the previous section, can be taken into account as each gain component is adapted separately. By imposing $G_{\theta}=\left[\begin{array}{cc}g_{\theta} & 0\end{array}\right], g_{\theta}>0$ and $G_{\omega}=\left[\begin{array}{cc}0 & g_{\omega}\end{array}\right], g_{\omega}<0, K_{1}(t) \equiv K_{\theta}(t)$ will always decrease when $\delta \theta$ is non-zero and $K_{2}(t) \equiv K_{\omega}(t)$ will always grow when $\delta \omega$ is non-zero. Combined with a modification which will be detailed in Section III.B, this will allow to have the gains evolve as required.

The second term in the adaptation law involving the $\Phi_{D_{j}, \alpha_{j}}$ function works as a barrier preventing the gains to escape some neighbourhood of the a priori given values of static gain $F_{0}$. More precisely, the following property is proved in. ${ }^{18}$

Lemma 1. If $e(t)$ is bounded for all $t \geq 0$ then $\left\|K_{j}(t)-F_{0 j}\right\|_{D_{j}}^{2}<\alpha_{j} \beta$ for all $t \geq 0$.

Besides guaranteeing that the gains will always stay bounded, the barrier function plays an important role in proving the stability of the adaptive algorithm. ${ }^{6}$ Indeed, the stability property is guaranteed by an appropriate choice for the parameters $\left(G_{j}, D_{j}, \alpha_{j}\right)_{j=1 \ldots \bar{\jmath}}$, where $G_{j}$ defines the first term used in the adaptation equation (11) and $D_{j}$ and $\alpha_{j}$ define the barrier function $\Phi_{D_{j}, \alpha_{j}}$. The following results give a strategy for finding such values, based on passivity properties of the closed-loop. The proofs are not reproduced here in order to give sufficient space for the application. Some elements for the proofs can be found in..$^{6,11}$ See also ${ }^{19}$ for the positive-real lemma on which following proposition is based:

Proposition 1. If $\dot{x}=A\left(F_{0}\right) x$ is asymptotically stable then there exist $\left(P, D_{j}, G_{j}\right), j=1 \ldots \bar{\jmath}$, solution to the following LMI problem: $P>\mathbf{0}$, 


$$
\left[\begin{array}{cccc}
\left\{P A\left(F_{0}\right)\right\}^{\mathcal{S}} & P B-C^{T} G_{1}^{T} & \cdots & P B-C^{T} G_{\bar{\jmath}}^{T} \\
B^{T} P-G_{1} C & -D_{1} & \cdots & \mathbf{0} \\
\vdots & \vdots & \ddots & \vdots \\
B^{T} P-G_{\bar{\jmath}} C & \mathbf{0} & \cdots & -D_{\bar{\jmath}}
\end{array}\right]<\mathbf{0} .
$$

Matrices $\left(G_{j}, D_{j}\right)$ then define weighted combinations of inputs $w$ and outputs $y=C x$ such that the following system is strictly passive:

$$
\left\{\begin{array}{l}
\dot{x}=A\left(F_{0}\right) x+B w \\
z=\left[\begin{array}{c}
G_{1} \\
\vdots \\
G_{\bar{\jmath}}
\end{array}\right] C x+\frac{1}{2}\left[\begin{array}{c}
D_{1} \\
\vdots \\
D_{\bar{\jmath}}
\end{array}\right] w
\end{array}\right.
$$

Theorem 1. Let $\beta>1$ be a given scalar. If $\left(F_{0}, G_{j}, D_{j}\right)_{j=1 \ldots j}$ is a feasible solution of the LMI problem (12), then there exist $\left(Q>0, R_{j}, T_{j}, F_{j}, \alpha_{j}\right)_{j=1 \ldots \bar{j}}$ solution to the following LMI problem:

$$
\begin{gathered}
{\left[\begin{array}{cc}
R_{j} & Q B-C^{T} G_{j}^{T} \\
B^{T} Q-G_{j} C & D_{j}
\end{array}\right] \geq \mathbf{0}, i=1 \ldots \bar{\imath}} \\
{\left[\begin{array}{cc}
T_{j} & \left(F_{j}-F_{0 j}\right)^{T} \\
\left(F_{j}-F_{0 j}\right) & D_{j}^{-1}
\end{array}\right] \geq \mathbf{0}, \operatorname{Tr}\left(T_{j}\right) \leq \alpha_{j}, j=1 \ldots \bar{\jmath}} \\
\left\{Q A\left(F_{0}\right)\right\}^{\mathcal{S}}+\sum_{j=1}^{\bar{j}}\left(\alpha_{j} \beta C_{j}^{T} C_{j}+R_{j}+\left\{C_{j}^{T}\left(F_{j}-F_{0 j}\right)^{T} G_{j} C\right\}^{\mathcal{S}}\right)<\mathbf{0 .} .
\end{gathered}
$$

The solution of the LMIs is such that $u=F$, where $F=\left[\begin{array}{lll}F_{1} & \ldots & F_{\bar{\imath}}\end{array}\right]$, is a stabilizing static output feedback verifying $\left\|F_{j}-F_{0 j}\right\|_{D_{j}}^{2} \leq \alpha_{j}$. Moreover, whatever positive definite $\Gamma_{j}>\mathbf{0}$, the parameters $\left(\Gamma_{j}, G_{j}, \alpha_{j}, D_{j}, \beta\right)_{j=1 \ldots j}$ define a stabilizing adaptive controller given by (11).

Based on these results, the synthesis procedure works as follows: starting from a known stabilizing static gain $F_{0}$, Proposition 1 combined to any additional linear constraints, allows to design by simple LMI solving some $\left(G_{j}, D_{j}\right)_{j=1 \ldots \bar{j}}$ matrices. These matrices being chosen, Theorem 1 outputs the set of scalars $\left(\alpha_{j}\right)_{j=1 \ldots \bar{j}}$ which are the last coefficients to be precisely chosen for guaranteeing stability of the adaptive control. The role of the scalars $\alpha_{j}$ is to parametrize the distance from the static gain $F_{0}$ that can take the adaptive gain $K(t)$ (see Lemma 1): the larger the $\alpha_{j}$, the more adaptation is allowed, without braking stability properties of the closed loop.

Since the sets $\left\{K_{j}:\left\|K_{j}-F_{0 j}\right\|_{D_{j}}^{2} \leq \alpha_{j} \beta\right\}$ not only depend of the values $\alpha_{j}$, but also on the values of $D_{j}$, a combined search of $\left(D_{j}, \alpha_{j}\right)$ is needed if one wants to maximize the sets of possible excursions of the gains $K_{j}$ around $F_{0 j}$. The following 2-step heuristic procedure is suggested when performing the design of the adaptive law:

1. Minimize $\sum_{j=1}^{\bar{j}} w_{j} \operatorname{Tr}\left(D_{j}\right)$ subject to (12) and other possible linear constraints on the $\left(G_{j}, D_{j}\right)$ matrices, were the $w_{j}>0$ are positive weights chosen depending on what parameters are expected to wary much or less.

2. Maximize $\sum_{j=1}^{\bar{\gamma}} w_{j} \alpha_{j}$ subject to (14-16).

Finally, ${ }^{18}$ suggests taking $\beta$ closed to 1 in order to limit conservatism of the LMIs and proposes the value $\beta=1.1$. This value is used throughout the results presented here.

The LMIs involved in the design procedure can be solved in polynomial time with efficient semi-definite programming algorithms such as SeDuMi. ${ }^{20}$ The results presented in the following sections have been coded with Matlab(c) by using the YALMIP interface. ${ }^{21}$

\section{III.B. The $\sigma$ - modification}

The $\sigma$-modification has been introduced since the first results on adaptive control in order to drive back the control gains to nominal values once the system has stabilized. It also plays a role in compensating persistent noise. For the considered structured adaptive laws, the $\sigma$-modification reads as follows:

$$
\dot{K}_{j}(t)=-G_{j} e e_{j}^{T} \Gamma_{j}-\sigma_{j}\left(K_{j}(t)-F_{0 j}\right) \Gamma_{j}-\Phi_{D_{j}, \alpha_{j}}\left(K_{j}(t)-F_{0 j}\right) \Gamma_{j}
$$


where the $\sigma_{j}$ are positive scalars. When neglecting the effects of the other terms, the $\sigma$-term drives the control gains to $F_{0 j}$ with first order linear dynamics. The larger is $\sigma_{j}$ the faster are these dynamics.

There exist few formal results for the stability of adaptive control under $\sigma$-modification. Convergence of the state to an equilibrium point is hard to prove in general; only results stating the converge to a bounded set are provided in. ${ }^{8}$ However, in our considered case, since the static gain $F_{0}$ - towards which the $\sigma$-term pushes the adaptive gain $K(t)$ is stabilizing, one can expect this modified law to guarantee asymptotic stability, at least locally. More precisely, let us consider the properties of the adaptive law depending on the values of $\sigma_{j}\left(K_{j}-F_{0 j}\right)$. When this term is negligible stability is then proved by the LMI conditions of Theorem 1 . When on the contrary $\sigma_{j}\left(K_{j}-F_{0 j}\right)$ is dominant then $K$ converges to $F_{0}$, which is a stabilizing static gain. The stability properties of the closed loop are therefore expected to be preserved.

The $\sigma$-modification makes the controller evolve between two values: the nominal gain $F_{0}$ and $K(t)$ - adaptive gain for large errors. This type of law is therefore useful for the satellite attitude control problem: for weak errors, the nominal behaviour is kept, with the controller varying little around $F_{0}$. When the errors are important, the system drifts from the nominal functioning point and $K(t)$ follows a law which can be approximated by (11), limiting the actuator solicitations. When the errors re-become weak, $K$ returns to $F_{0}$ and the nominal behaviour is recovered.

\section{III.C. Discrete implementation of the adaptive law}

In view of implementation, the algorithm (17) is sampled, based on the Euler transformation $\left(\mathrm{see}^{22,23}\right)$. The barrier function is replaced by a projector whose effect is illustrated, for a two-dimensional case, on Figure 3: if the computed gain $\tilde{K}_{j}$ is such that $\left\|\tilde{K}_{j}-F_{0 j}\right\|_{D_{j}}^{2} \geq \alpha_{j} \beta$, then $\tilde{K}_{j}$ is projected on the domain border $\left\|K_{j}-F_{0 j}\right\|_{D_{j}}^{2}=\alpha_{j} \beta$, along the line connecting $\tilde{K}_{j}$ to $F_{0 j}$.

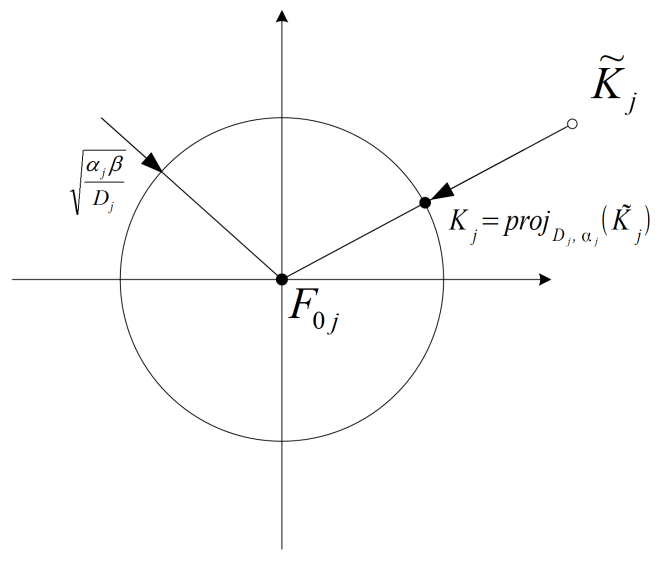

Figure 3. Projector function

The following adaptive algorithm is thus proposed:

$$
\left\{\begin{array}{l}
\tilde{K}_{j}(k)=K_{j}(k-1)-G_{j} e(k) e_{j}(k)^{T} \Gamma_{j} T_{s}-\sigma_{j}\left(K_{j}(k-1)-F_{0}\right) \Gamma_{j} T_{s} \\
K_{j}(k)=\operatorname{proj}_{D_{j}, \alpha_{j}}\left(\tilde{K}_{j}(k), F_{0}\right) \\
u(k)=\sum_{j=1}^{\bar{j}} K_{j}(k) e_{j}(k)
\end{array}\right.
$$

where $T_{s}$ is the sampling period.

Remark: If the gain $K_{j}$ is scalar, the projector can be expressed as a saturation function:

$$
\left.\operatorname{proj}_{D_{j}, \alpha_{j}}\left(\tilde{K}_{j}, F_{0 j}\right)\right|_{\tilde{K}_{j} \in \mathrm{R}}=\operatorname{sat}_{D_{j}, \alpha_{j}}\left(\tilde{K}_{j}, F_{0 j}\right)= \begin{cases}\tilde{K} & , \text { if } F_{0}-\sqrt{\frac{\alpha_{j} \beta}{D_{j}}} \leq \tilde{K} \leq F_{0}+\sqrt{\frac{\alpha_{j} \beta}{D_{j}}} \\ F_{0}-\sqrt{\frac{\alpha_{j} \beta}{D_{j}}}, & \text { if } \tilde{K}<F_{0}-\sqrt{\frac{\alpha_{j} \beta}{D_{j}}} \\ F_{0}+\sqrt{\frac{\alpha_{j} \beta}{D_{j}}}, & \text { if } \tilde{K}>F_{0}+\sqrt{\frac{\alpha_{j} \beta}{D_{j}}}\end{cases}
$$




\section{Adaptive control for DEMETER}

The adaptive control strategy exposed in Section III is now applied for the satellite. The general methodology is to replace the six static gains $\left(K_{\theta \mathrm{i}}, K_{\omega \mathrm{i}}\right)_{\mathrm{i} \in\{\mathrm{x}, \mathrm{y}, \mathrm{z}\}}$ with adaptive gains. Preliminary results for only one axis control, with unstructured adaptive control and without $\sigma$-modification have been published in. ${ }^{6}$ Here we consider the 3-axes case and improve the results thanks to the structured adaptive control and do not deteriorate the optimality for low pointing errors with an appropriate usage of the $\sigma$-modification.

\section{IV.A. Design of the adaptive control law}

The synthesis model of each axis is given by:

$$
\left[\begin{array}{c}
1 \\
H_{\text {estim }}(s)
\end{array}\right] H_{\text {sat }, \mathrm{i}}(s) H_{\mathrm{RW}}(s) H_{\text {filter }, \mathrm{i}}(s), \mathrm{i} \in\{\mathrm{x}, \mathrm{y}, \mathrm{z}\}
$$

The state space matrices $\left(A_{\mathrm{i}}, B_{\mathrm{i}}, C_{\mathrm{i}}\right)$ are then obtained and the 2-step design procedure described in Section III.A is applied three times, once for each axis.

For the first step, solving the LMIs (12), the constraints $G_{\omega \mathrm{i}}=\left[\begin{array}{ll}0 & g_{\omega \mathrm{i}}\end{array}\right], g_{\omega \mathrm{i}}<0$ and $G_{\theta \mathrm{i}}=\left[\begin{array}{ll}g_{\theta \mathrm{i}} & 0\end{array}\right]$, $g_{\theta \mathrm{i}}>0$ are added such that the gains evolve according to the specifications described in Section II.D.

The continuous-time adaptation law for each scalar gain reads:

$$
\begin{aligned}
& \dot{K}_{\theta \mathrm{i}}(t)=-\left(g_{\theta \mathrm{i}} \delta \theta_{\mathrm{i}}^{2}+\sigma_{\theta \mathrm{i}}\left(K_{\theta \mathrm{i}}(t)-F_{0 \theta}\right)+\Phi_{D_{\theta \mathrm{i}}, \alpha_{\theta \mathrm{i}}}\left(K_{\theta \mathrm{i}}(t)-F_{0 \theta}\right)\right) \Gamma_{\theta \mathrm{i}} \\
& \dot{K}_{\omega \mathrm{i}}(t)=-\left(g_{\omega \mathrm{i}} \delta \omega_{\mathrm{i}}^{2}+\sigma_{\omega \mathrm{i}}\left(K_{\omega \mathrm{i}}(t)-F_{0 \omega}\right)+\Phi_{D_{\omega \mathrm{i}}, \alpha_{\omega \mathrm{i}}}\left(K_{\omega \mathrm{i}}(t)-F_{0 \omega}\right)\right) \Gamma_{\omega \mathrm{i}}
\end{aligned}, \mathrm{i} \in\{\mathrm{x}, \mathrm{y}, \mathrm{z}\}
$$

This gives the following discrete adaptive algorithm:

$$
\begin{aligned}
& \tilde{K}_{\theta \mathrm{i}}(k)=K_{\theta \mathrm{i}}(k-1)-\left(g_{\theta \mathrm{i}} \cdot \delta \theta_{\mathrm{i}}^{2}(k)+\sigma_{\theta \mathrm{i}}\left(K_{\theta \mathrm{i}}(k-1)-F_{0 \theta}\right)\right) \Gamma_{\theta \mathrm{i}} T_{s} \\
& \tilde{K}_{\omega \mathrm{i}}(k)=K_{\omega \mathrm{i}}(k-1)-\left(g_{\omega \mathrm{i}} \cdot \delta \omega_{\mathrm{i}}^{2}(k)+\sigma_{\omega \mathrm{i}}\left(K_{\omega \mathrm{i}}(k-1)-F_{0 \omega}\right)\right) \Gamma_{\omega \mathrm{i}} T_{s} \\
& K_{\theta \mathrm{i}}(k)=\operatorname{sat}_{D_{\theta \mathrm{i}}, \alpha_{\theta \mathrm{i}}}\left(\tilde{K}_{\theta \mathrm{i}}(k), F_{0 \theta}\right) \\
& K_{\omega \mathrm{i}}(k)=\operatorname{sat}_{D_{\omega \mathrm{i}}, \alpha_{\omega \mathrm{i}}}\left(\tilde{K}_{\omega \mathrm{i}}(k), F_{0 \omega}\right) \\
& T_{a \mathrm{i}}(k)=-\left(K_{\theta \mathrm{i}}(k) \cdot \delta \theta_{\mathrm{i}}(k)+K_{\omega \mathrm{i}}(k) \cdot \delta \omega_{\mathrm{i}}(k)\right) \\
& T_{p \mathrm{i}}(k)=H_{\text {filter }, \mathrm{i}}(z) T_{a \mathrm{i}}(k)
\end{aligned}
$$

for $\mathrm{i} \in\{\mathrm{x}, \mathrm{y}, \mathrm{z}\}$. The sampling period $T_{s}=0.25 \mathrm{~s}$ in the case of the Myriade AOCS and $H_{\text {filter, }}(z)$ are the discrete transfer functions obtained from the continuous-time filters $H_{\text {filter }, \mathrm{i}}(s)$ by applying the bilinear transformation. The control structure of each axis thus contains a proportional-derivative adaptive controller (22-26), with gains adapted around the nominal values $F_{0 \theta}=0.1, F_{0 \omega}=2$. This adaptive structure is followed by the invariant filter (22).

The values of the different parameters, obtained by solving the LMIs (12-16), are summarized in Table 1 . The weights $w_{\theta}=100, w_{\omega}=1$ have been chosen in order to give more importance to large variation domains on the $K_{\theta \mathrm{i}}$ gains. The domains inside which the gains for each axis are allowed to evolve are given in Table 2. There are almost $100 \%$ admissible variations of the adapted $K_{\theta \mathrm{i}}$ gains and about $20 \%$ admissible variations of the adapted $K_{\omega \mathrm{i}}$ gains.

Table 1. LMI solving results

\begin{tabular}{|c|c|c|c|c|c|c|}
\hline & $g_{\theta \mathrm{i}}$ & $g_{\omega \mathrm{i}}$ & $D_{\theta \mathrm{i}}$ & $D_{\omega \mathrm{i}}$ & $\alpha_{\theta \mathrm{i}}$ & $\alpha_{\omega \mathrm{i}}$ \\
\hline \hline x axis & 53.52 & -941.44 & 1135.46 & 9683.27 & 8.9 & 1831 \\
\hline y axis & 175.71 & -208.04 & 3615.9 & 30954 & 28.56 & 3370.7 \\
\hline z axis & 231.04 & -843.44 & 4754.61 & 40796.6 & 36.17 & 4338.59 \\
\hline
\end{tabular}

Another important choice is that of $\sigma_{\theta \mathrm{i}}$ and $\sigma_{\omega \mathrm{i}}$. The following reasoning illustrates, how this choice is made for the parameters $\sigma_{\theta i}$ : suppose a large attitude reference is applied, for instance, on the $\mathrm{X}$ axis. After this, the large value of the $\delta \theta_{\times}$term in the adaptation equation (22) causes the gain $K_{\theta \times}$ to decrease and reach the border of the variation domain. At this point its value is constant: $K_{\theta \mathrm{x}}=K_{\theta \times, b}=0.0071$, as the saturation function (24) stops the gain from 
Table 2. LMI solving results

\begin{tabular}{|c|c|c|c|}
\hline & x axis & $y$ axis & $z$ axis \\
\hline \hline$K_{\theta \mathrm{i}}$ & {$\left[\begin{array}{ll}0.0071 & 0.1929\end{array}\right]$} & {$\left[\begin{array}{ll}0.0072 & 0.1927\end{array}\right]$} & {$\left[\begin{array}{ll}0.0072 & 0.1927\end{array}\right]$} \\
\hline$K_{\omega \mathrm{i}}$ & {$\left[\begin{array}{ll}1.5439 & 2.4561\end{array}\right]$} & {$\left[\begin{array}{ll}1.6580 & 2.3927\end{array}\right]$} & {$\left[\begin{array}{ll}1.6539 & 2.3461\end{array}\right]$} \\
\hline
\end{tabular}

decreasing further. $K_{\theta \mathrm{x}}$ will then leave the domain limit and evolve towards the value $F_{0 \theta}=0.1$ when the $\sigma$-term balances the error term. This occurs when the attitude error reaches a threshold value, $\delta \theta_{\text {thresh }, x}$, verifying:

$$
-g_{\theta \mathrm{x}} \cdot \delta \theta_{\mathrm{thresh}, \mathrm{x}}^{2}=\sigma_{\theta \mathrm{x}}\left(K_{\theta \mathrm{x}, b}-F_{0 \theta}\right)
$$

As $g_{\theta \mathrm{x}}, K_{\theta \mathrm{x}, b}$ and $F_{0 \theta}$ are known, the parameter $\sigma_{\theta \mathrm{x}}$ allows defining the point at which the gain returns to its nominal value. In the following simulations, two sets of numerical values are used: $\left\{\sigma_{\theta \mathrm{x}}=4.4, \sigma_{\theta \mathrm{y}}=14.4, \sigma_{\theta \mathrm{z}}=19\right\}$ and $\left\{\sigma_{\theta \mathrm{x}}=1.1, \sigma_{\theta \mathrm{y}}=3.6, \sigma_{\theta \mathrm{z}}=4.8\right\}$ corresponding to thresholds of 5 and 2.5 degrees respectively for each axis.

The same reasoning applies for choosing the $\sigma_{\omega i}$ values. The choices $\sigma_{\omega x}=5.66 \cdot 10^{-4}, \sigma_{\omega y}=1.65 \cdot 10^{-4}$ and $\sigma_{\omega z}=6.76 \cdot 10^{-4}$ are made, which correspond to a speed threshold of $0.03 \mathrm{deg} / \mathrm{s}$ for each axis.

The last parameters to be chosen are the $\Gamma_{\theta i}$ and $\Gamma_{\omega i}$ adaptation gains. The simulations are done with the following values

$$
\begin{aligned}
& \Gamma_{\theta \mathrm{x}}=0.15 \quad, \quad \Gamma_{\theta \mathrm{y}}=0.05 \quad, \quad \Gamma_{\theta \mathrm{z}}=0.03 \\
& \Gamma_{\omega x}=9.7 \quad, \quad \Gamma_{\omega y}=43.9 \quad, \quad \Gamma_{\omega z}=10.8
\end{aligned}
$$

that have been chosen in order to have reasonable values of the gains variation, $K_{\theta \mathrm{i}}(k)-K_{\theta \mathrm{i}}(k-1)$ and $K_{\omega \mathrm{i}}(k)-$ $K_{\omega i}(k-1)$, over the whole functioning domain.

\section{IV.B. Simulation results}

Simulation results corresponding to the satellite in closed loop configuration with the adaptive law (22-27) are given next. A 3-axes simulator of the DEMETER AOCS, involving the flexible satellite, actuators and sensors models is considered. The satellite model with the inertias having nominal values and flexible modes defined by (3) is first considered. Responses obtained with the adaptive law with two sets of values for the $\sigma_{\theta}$ parameters are compared with results given by the application of the existing control strategy, based on the switching law (5-6), described in Section II.C,

Figures 4 and 5 show the satellite response to step attitude references of amplitude of 20, 10 and 15 degrees for the $x, y$ and $z$ axis respectively. The first step reference covers the whole domain of the Demeter mission mode. Figure 4 shows the signals corresponding to the $\mathrm{X}$ axis: measured satellite angular position and speed, the corresponding adaptive gains as well as the actuator response (angular reaction wheel speed). The same signals corresponding to the $y$ and $z$ axes are plotted in Figure 5.

These results show that the proportional-derivative adaptive law allows tracking of attitude references of large values without saturating the actuators: even if the adaptive law generates larger a control action than the switch-based law, the angular speeds attained by the reaction wheels do not surpass $40 \%$ of the maximal allowed value. The adaptation of the gains thus allows to extend significantly the functioning domain which could be covered by the static proportional-derivative law. The switch-based law could therefore be replaced by the proposed adaptive algorithm: this would also allow a gain in response time, as it can be seen on the attitude plots of Figures 4 and 5.

When analysing the plots of the adaptive gains, three phases can be observed: first, after the reference is applied the gains evolve rapidly and reach the barrier of their allowed variation domains. Follows a phase when they remain constant, at values on the barrier (for the $\mathrm{x}$ axis, this occurs for $K_{\theta \times}$ between $t=0$ and $t \approx 500$, and for $K_{\omega \times}$ between $t=0$ and $t \approx 250$ ). Finally, the gains return to their nominal values once that the attitude error and the angular speed respectively are lower than the thresholds defined by the values of the $\sigma$ parameters.

The influence of these parameters is easy to observe. On the $\mathrm{x}$ axis for instance, for $\sigma_{\theta \times}=4.4, K_{\theta \times}$ starts evolving towards its nominal value when $\delta \theta_{\mathrm{x}}=5 \operatorname{deg}(t \approx 500 s)$, while if $\sigma_{\theta \mathrm{x}}=1.1, K_{\theta \mathrm{x}}$ returns to its nominal value when $\delta \theta_{\mathrm{x}}=2.5 \mathrm{deg}(t \approx 700 \mathrm{~s})$, as predicted by $(28)$. When $K_{\theta \mathrm{x}}$ starts increasing an acceleration phase can be observed on the attitude plots. The moment when this acceleration appears thus depends on the value of $\sigma_{\theta \mathrm{x}}$ and this parameter influences in this way the response time. For $\sigma_{\theta \mathrm{x}}=4.4$ the acceleration phase occurs earlier $(t \approx 500 s)$ than for $\sigma_{\theta \mathrm{x}}=1.1(t \approx 700 \mathrm{~s})$ and thus the response is faster when $\sigma_{\theta \mathrm{x}}$ is larger. At the same time, one can also note that the actuator response is also of larger amplitude for larger values of $\sigma_{\theta x}$. This analysis shows that the values of $\sigma$ influence the closed loop behaviour of the satellite. $\sigma_{\theta i}$ in particular can thus be used as design parameters. 

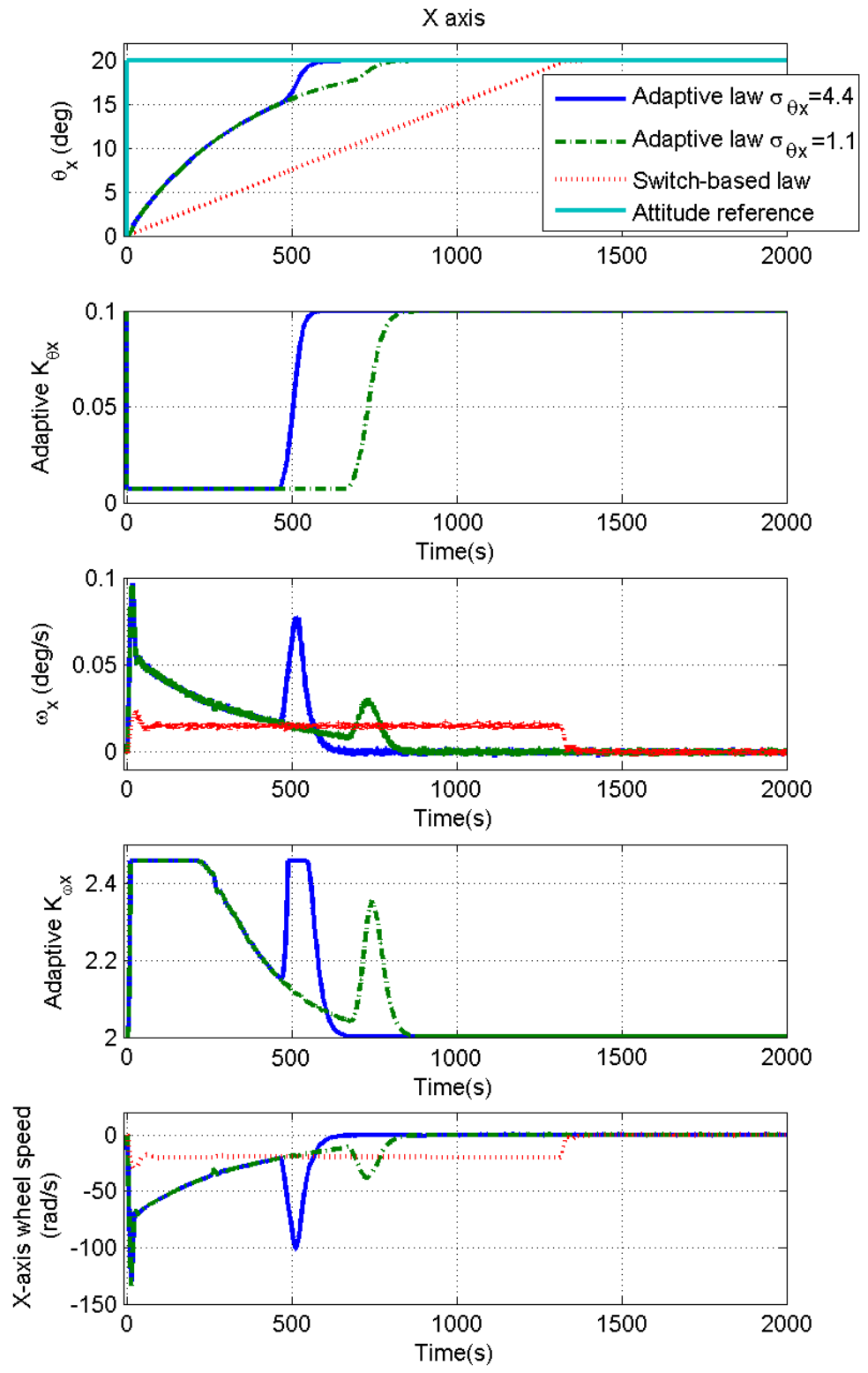

Figure 4. Tracking of attitude references - $\mathrm{X}$ axis 

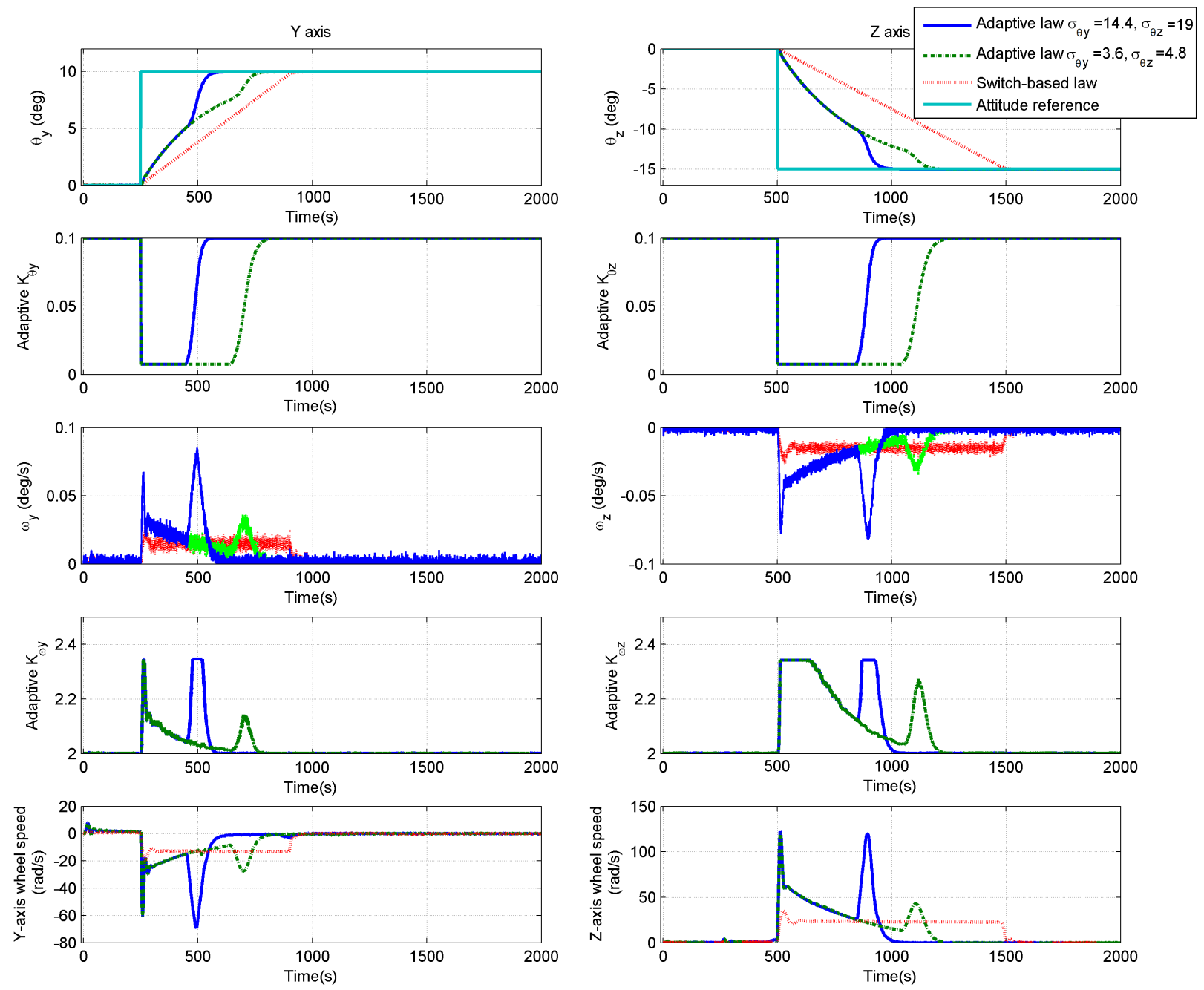

Figure 5. Tracking of attitude references $-Y$ and $Z$ axis 


\section{Robustness tests}

Results of robustness tests of the adaptive law are now presented. First consider the robustness with respect to parametric uncertainties. The previous results considered a simulation model containing four flexible modes, where the satellite inertia $J$ was equal to its nominal value. The case where the satellite inertia $J$ has different values is now considered.

The uncertainty on this parameter was estimated at $20 \%$ of the nominal value and several cases were tested for $J$ having values between $J_{s}=0.8 \cdot J_{n o m}$ and $J_{b}=1.2 \cdot J_{n o m}$, where $J_{n o m}$ is the nominal value of the inertia matrix. The adaptive law is able to handle these uncertainties and the output responses are very close to the ones obtained with the nominal inertia value. As they are very similar to the previous figures, they are not represented here. The actuator responses however are significantly different and are represented in Figure 6 for the adaptive law with $\left\{\sigma_{\theta \mathrm{x}}=4.4, \sigma_{\theta \mathrm{y}}=14.4, \sigma_{\theta \mathrm{z}}=19\right\}$. Two responses are plotted, for the value of the inertia at the extremity of the uncertainty interval: $J=J_{s}=0.8 \cdot J_{\text {nom }}$ and $J=J_{b}=1.2 \cdot J_{\text {nom }}$. With respect to the nominal case a smaller control action can be observed for $J=J_{s}$. On the contrary, when $J=J_{b}$, the actuator response is of larger amplitude, remaining however far from the saturation value of $293 \mathrm{rad} / \mathrm{s}$.
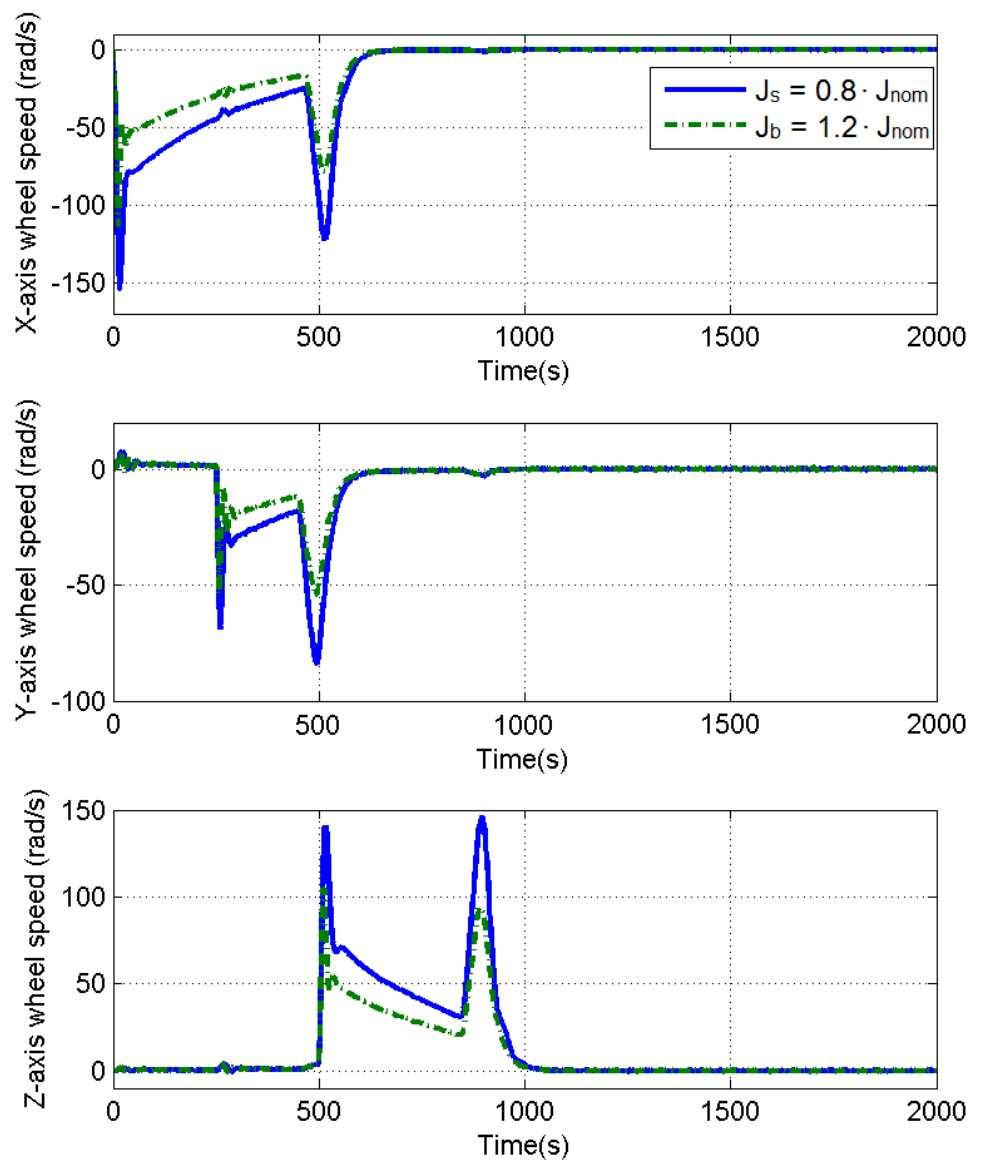

Figure 6. Reaction wheels response for different values of the inertia

The influence of measurement noise on the adaptive closed-loop system is now analysed. The following simulations consider the case where the satellite is in steady-state and the attitude reference is equal to zero. In the presence of noise the tracking errors for the satellite's attitude and speed are never identically zero. As the error terms used in the adaptation equations (22-23) contain the squares of these errors, the gains are expected to drift in a direction defined by the sign of $g_{\theta \mathrm{i}}$ and $g_{\omega \mathrm{i}}$ respectively. This drift is balanced by the $\sigma$-terms which act as a low-pass filters and push the gains back to their nominal values. Even if the drift is balanced, the equilibrium point $K_{\theta \mathrm{i}}=F_{0 \theta}, K_{\omega \mathrm{i}}=F_{0 \omega}$ is lost and an offset with respect to $F_{0}$, along with transmission of the noise, is expected to appear on the adaptive gains.

This behaviour is illustrated in Figure 9, where the adaptive gains for the $\mathrm{z}$ axis are plotted, similar responses being 
obtained for the other axes. The gains stay very close to their nominal values, $\{0.1,2\}$ and as the measurement noise is augmented by the speed estimator (high-pass filter), $K_{\omega}$ suffers more from the disturbance. The offset on this gain is however of less than $0.1 \%$ with respect to its nominal value.
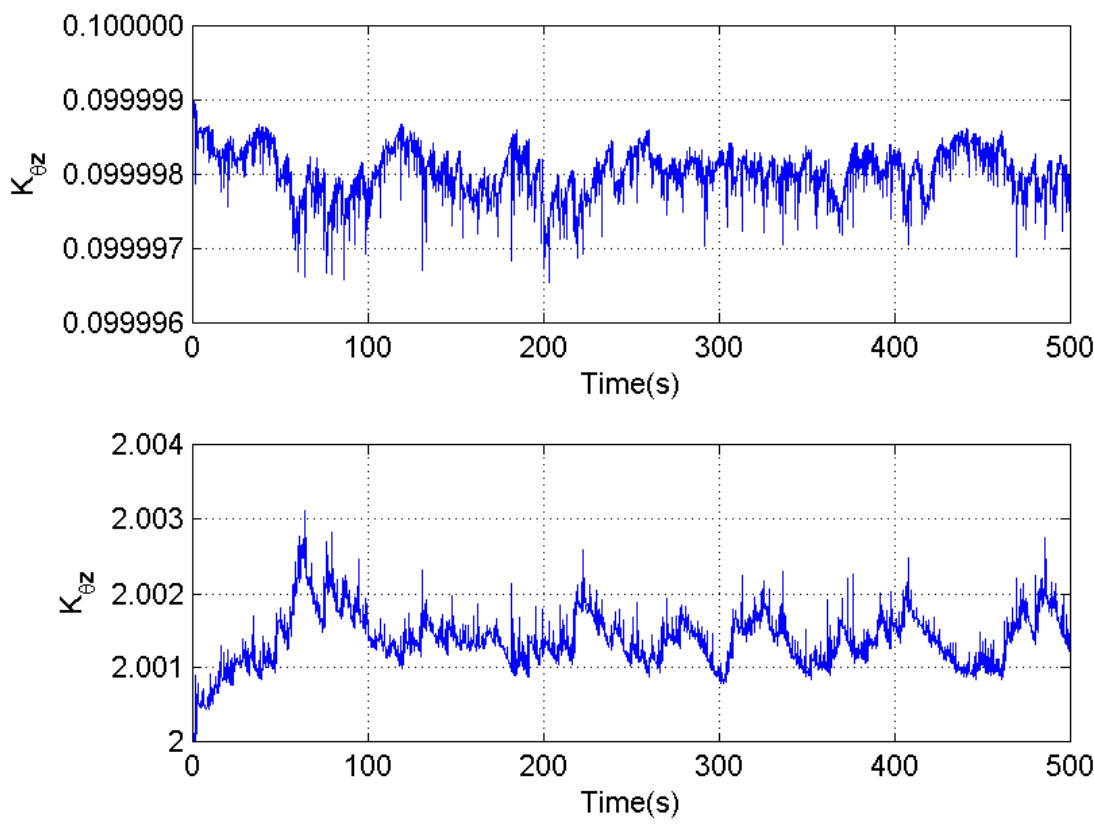

Figure 7. Influence of measurement noise on the adaptive gains - $\mathrm{Z}$ axis

The computed torque $T_{a z}$ obtained in steady-state with the switch-based law and with the adaptive law respectively is shown in Figure 8. Note that for the switch-based law only the proportional-derivative branch (6) having constant gains is active, as the pointing error is much smaller than the threshold $\theta_{L}=0.3 \mathrm{deg}$. The influence of noise can be seen on both control signals and it can be noticed that the noise levels are the same in both cases. Thus, even though the adaptive gains are influenced by the measurement noise, the impact on the computed control signal is negligible with respect to the case where the gains are constant. The optimal behaviour guaranteed at low angles by the static controller is thus preserved with the adaptive law.
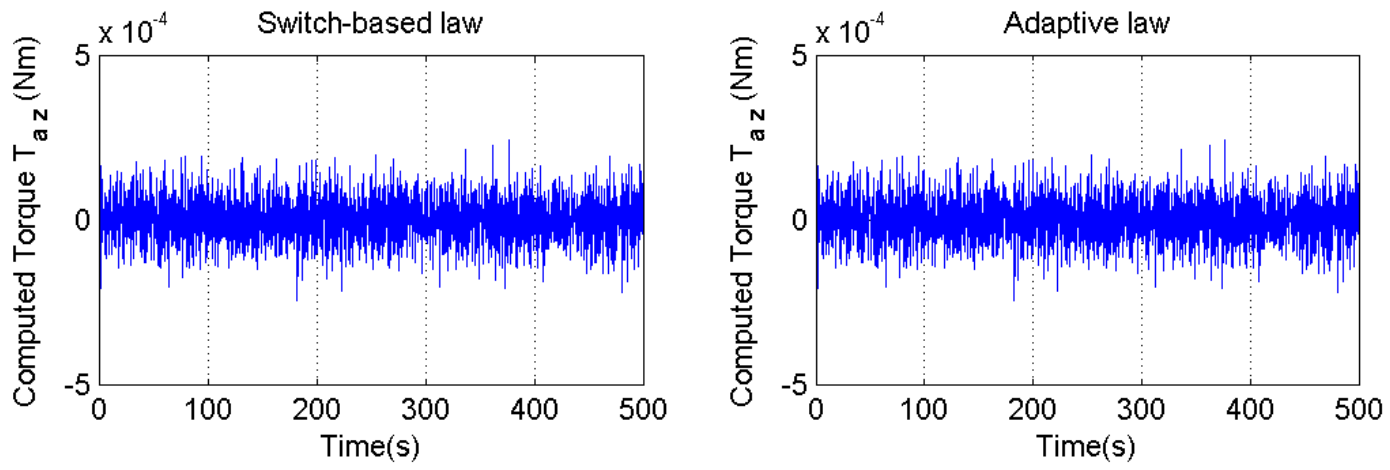

Figure 8. Influence of measurement noise on computed torque - adaptive and switch-based law - $\mathrm{Z}$ axis

\section{Conclusions}

Motivated by the need of having satellite attitude controllers able to cover enlarged operating domains, a new proportional-derivative adaptive control algorithm was developed. Starting from a known static controller, the presented synthesis technique allowed obtaining an adaptive control law which in the case of Myriade satellite can be used over the whole mission mode. 

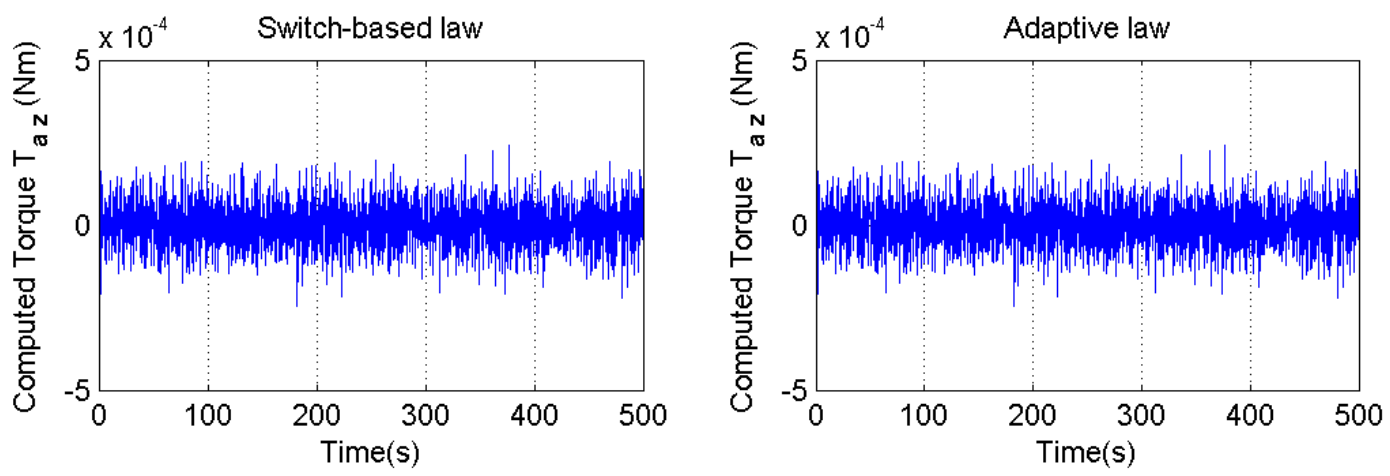

Figure 9. Influence of measurement noise on computed torque - adaptive and switch-based law - $\mathrm{Z}$ axis

The new adaptive algorithm has a discrete-time expression and uses only the measured outputs of the system, not needing any additional excitation. The required computational effort is limited,making the adaptive algorithm suitable for implementation on on-board satellite computers. The simulation results confirmed the interest of using an adaptive control law: step references of up to 20 degrees can be tracked without engendering the saturation of the reaction-wheel, while model uncertainties as well as persisting noise can be coped with. Finally, the adaptive algorithm disposes of several design parameters and a connection between the values of $\sigma_{\theta \mathrm{i}}$ and the response time for each axis was shown.

Based on these preliminary positive results, in-flight tests on the Picard satellite are being considered.

\section{References}

${ }^{1}$ Buisson, F., "The DEMETER program: a pathfinder to a high performance microsatellite line," AIAA USU conference on small satellites, Logan, Utah, USA, 2003, pp. 441-446.

${ }^{2}$ T. Cussac, et al., "The Demeter microsatellite and ground segment," Planetary and Space Science, Vol. 54, No. 5, 2006, pp. $413-427$.

${ }^{3}$ Pittet, C. and Arzelier, D., "DEMETER: a benchmark for robust analysis and control of the attitude of flexible microsatellites," IFAC Symposium on Robust Control Design, Toulouse, France, 2006.

${ }^{4}$ Pittet, C., Mignot, J., and Fallet, C., "LMI based multi-objective $H_{\infty}$ control of flexible microsatellites," IEEE Conference on Decision and Control, Sydney, Australia, 1999.

${ }^{5}$ Peaucelle, D., Bortott, A., Gouaisbaut, F., Arzelier, D., and Pittet, C., "Robust analysis of DEMETER benchmark via quadratic separation," IFAC Symposium on Automatic Control in Aerospace, Nara, Sept. 2010.

${ }^{6}$ Peaucelle, D., Drouot, A., Pittet, C., and Mignot, J., "Simple Adaptive Control without Passivity Assumptions and Experiments on Satellite Attitude Control DEMETER Benchmark," IFAC World Congress, Aug. 2011.

${ }^{7}$ Fradkov, A., "Adaptive Stabilization of a Linear Dynamic Plant," Autom. Remote Contr., Vol. 35, No. 12, 1974, pp. $1960-1966$.

${ }^{8}$ Kaufman, H., Barkana, I., and Sobel, K., Direct adaptive control algorithms, Springer, New York, 1998.

${ }^{9}$ Peaucelle, D., Fradkov, A., and Andrievsky, B., "Passification-Based Adaptive Control of Linear Systems: Robustness Issues," Int. J. of Adaptive Control and Signal Processing, Vol. 22, No. 6, Aug. 2008, pp. 590-608, doi: 10.1002/acs.1009.

${ }^{10}$ Fradkov, A. and Andrievsky, B., "Passification-based adaptive control with implicit reference model," alcsop, St. Petersburg, 2007.

${ }^{11}$ Peaucelle, D., Andrievsky, B., Mahout, V., and Fradkov, A., "Robust Simple Adaptive Control with Relaxed Passivity and PID control of a Helicopter Benchmark," IFAC World Congress, 2011.

${ }^{12}$ Fradkov, A., "Passification of Non-square Linear Systems and Feedback Yakubovich-Kalman-Popov Lemma," European J. of Control, Vol. 6, 2003, pp. 573-582.

${ }^{13}$ Barkana, I., "Gain conditions and convergence of simple adaptive control," International Journal of Adaptive Control and Signal Processing, Vol. 19, No. 1, 2005, pp. 13-40.

${ }^{14}$ Manceaux-Cumer, C., "Minimal LFT form of a spacecraft built up from two bodies," AIAA Guidance, Navigation and Control Conference., Montreal, Canada, 2001.

${ }^{15}$ Tarbouriech, S., Garcia, G., Gomes da Silva Jr, J.-M., and Queinnec, I., Stability and Stabilization of Linear Systems with Saturating Actuators, Springer, 2011.

${ }^{16}$ Biannic, J.-M. and Tarbouriech, S., "Optimization and implementation of dynamic anti-windup compensators in aircraft control systems with multiple saturations." Control Engineering Practice, Vol. 17, No. 6, 2009, pp. 703-713.

${ }^{17}$ Zaccarian, L. and Teel, A., Modern Anti-windup Synthesis: Control Augmentation for Actuator Saturation, Princeton Series in Applied Mathematics, Princeton University Press, 2011.

${ }^{18}$ Peaucelle, D. and Fradkov, A., "Robust adaptive $L_{2}$-gain control of polytopic MIMO LTI systems - LMI results," Systems \& Control Letters, Vol. 57, No. 11, 2008, pp. 881-887.

${ }^{19}$ Boyd, S., Ghaoui, L. E., Feron, E., and Balakrishnan, V., Linear Matrix Inequalities in System and Control Theory, SIAM Studies in Applied Mathematics, Philadelphia, 1994. 
${ }^{20}$ Sturm, J., "Using SeDuMi 1.02, a MATLAB toolbox for optimization over symmetric cones," Optimization Methods and Software, Vol. 1112, 1999, pp. 625-653.

${ }^{21}$ Löfberg, J., YALMIP : A Toolbox for Modeling and Optimization in MATLAB, 2004.

${ }^{22}$ R. Ben Yamin, I.Yaesh, U., "Robust Discrete-Time Simple Adaptive Tracking," Mediteranean Conference on Control and Automation, Athens, 2007.

${ }^{23}$ Barkana, I., "On Stability and Gain Convergence in Discrete Simple Adaptive Control," IFAC World Congress, Prague, 2005. 